\title{
A Study of Bio-Inspired Jumping Robots
}

\author{
Kanwalpreet Kaur \\ Assistant Professor Guru Nanak College \\ Budhlada, Punjab, India
}

\author{
Gurpreet Singh \\ Assistant Professor Guru Nanak College \\ Budhlada, Punjab, India
}

\begin{abstract}
Many tiny robots have developed which walk in smooth surface but the problem face in complex terrain. Some wheel based robot move in flat surface only. In this paper, we studied some bio-inspired jumping robots like Grillo, MiniWhegs, MSU jumper, EPFL. Because Jumping is used by small animals in nature, rough terrain and cluttered environment. These robots use energy store and release mechanism.
\end{abstract}

\section{General Terms}

Jumping robots, self-righting, steering, terrain.

\section{Keywords}

Bio-inspired jumping robot,. self-righting

\section{INTRODUCTION}

In the natural world, different jumping mechanisms have been evolved by a great number of organisms, from little insects like fleas to big animal like kangaroo. This locomotion strategy has been used mainly to increase the forward speed, or to escape from predators in a more efficient way, or to launch into flight [3]. We already describe there are certain problem face by robots to move in uncertain path. To overcome this problem the scientist accept the jumping locomotion rather than ground locomotion. Locomotion is a key issue for autonomous robots, especially if considering efficiency in micro-robots, which can be adopted for exploration and monitoring in unstructured environments. Despite the mechanical and kinematic complication, legged locomotion presents several advantages in unstructured or uneven terrains[2].

\section{MSU JUMPER}

This is the Michigan State University jumper robot made by Jianguo Zhao. The robot has a dimension $6 \mathrm{~cm} \times 8 \mathrm{~cm} \times 2 \mathrm{~cm}$ and weighs 20 grams. In this robot to achieve the continues jumping several mechanism used like energy store and release mechanism, self-righting mechanism, and jumping direction changing mechanism. This robot can jump $55 \mathrm{~cm}$ with a $75^{\circ}$ takeoff angle [1]. There are many constrains in case of jumping is size and weight. So, This robot need single motor and weight will not increase.

The mechanism of store energy and release is best. Steering mechanism used for speed reduction, which is new. It can change its orientation, right itself, and then jump. The actual jumping mechanism was directly inspired by the legs of a frog. Jumping principle is based to the springs.

\subsection{MSU JUMPER WORKING}

- The pager motor gear pulls the robot's body down towards its legs and slowly charging four springs. The gearing and springs help keep the power requirements low without sacrificing jumping energy. When the springs are fully charged up, the gear trips a little lever, and the legs are released(figure1). After that the robot finds itself flying mode(figure 2).

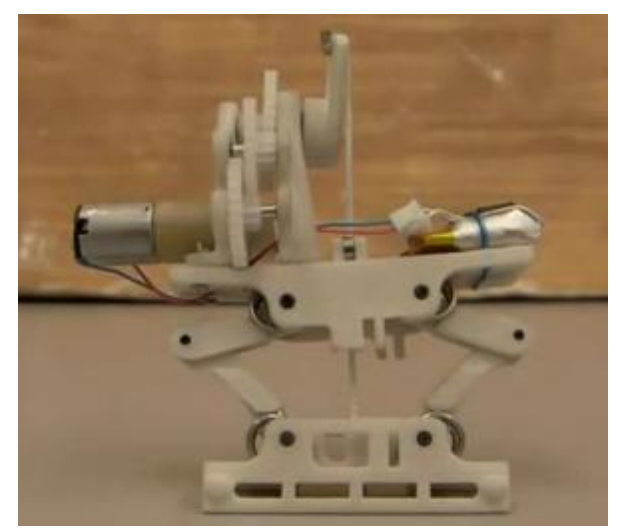

Figure 1: pull down body towards legs

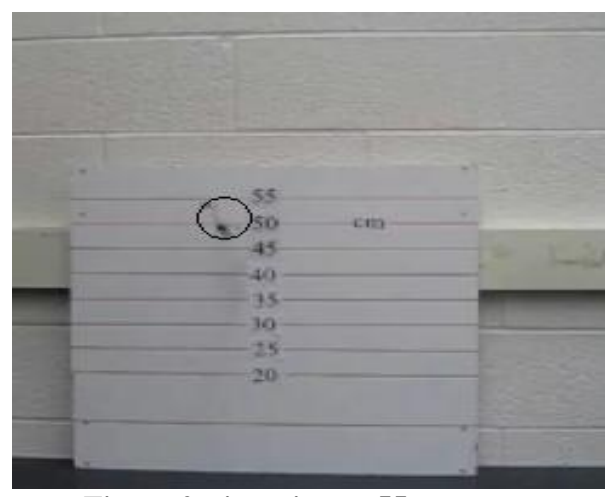

Figure 2: jumping at $55 \mathrm{~cm}$

- Then the paper motor driving backwards, the gear that charges the springs instead spins against the ground without engaging anything, allowing the body of the robot to rotate to a new position (figure 3,4).

- To stand up, as the robot's body pulls down towards its legs, little arms stretch outwards(figure 5).and ready to take the another jump.

MSU jumper robot can be used as mobile sensors and in rough terrain and natural obstacles which are not suitable for sensors with wheels[1]. 


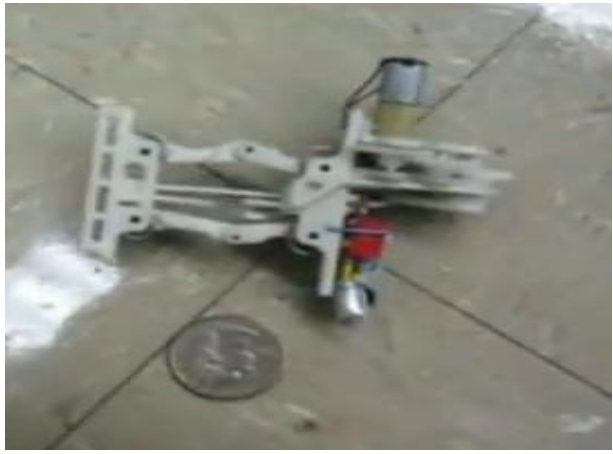

Figure 3: Steering in one direction

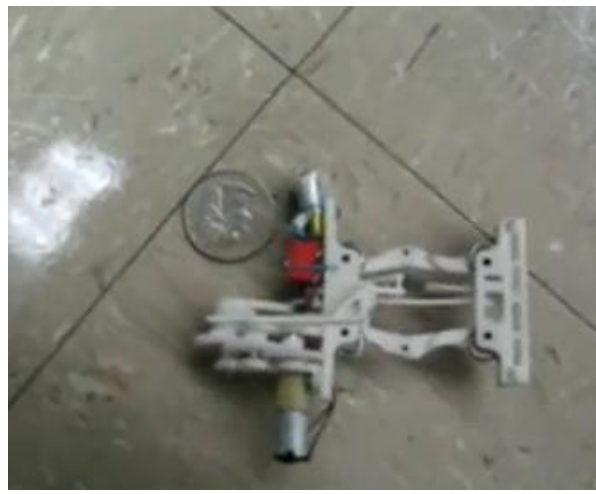

Figure 4: steering in other direction

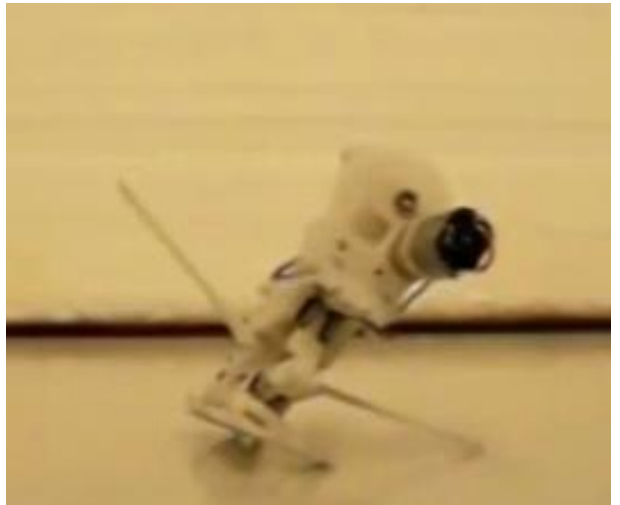

Figure5: Self righting

\section{GRILLO II}

Grillo II is improving robot of Grillo. To represent the optimum design of jumping, mapping force into constant force at foot-ground interface. Grillo II inspired from the leafhopper after observing the jumping mechanism. Insect observation of legs morphology and kinematics, as well as internal mechanisms for storage and release of muscular power. The observers looked that Cicadellidae, Hemiptera was not losing the contact to ground of hind legs before jumping[5].

\subsection{WORKING OF GRILLO II}

"GRILLO II", 0.3 watt DC motor is used as the energy source to load a spring, storing jumping energy. After storing enough energy, it release it like above robot. The rear legs used for generating the jumping innovative improvements . Due to a variable gear-ratio property imposed by the leg kinematics, both motion obit of the feet and dynamic characteristics of the jumping are very similar to insects.

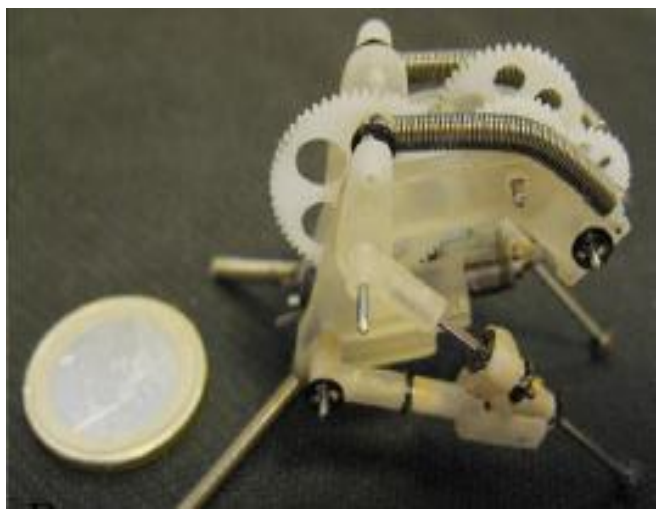

Figure 6 Grillo II

\section{EPFL}

There are so many challenges in jumping robot. As the size of robot decrease then the obstacles will also increase more then to large robot. École Polytechnique Fédérale de Lausanne (EPFL) present three robotic jumpers that solve those challenges and develop to solve the difficulties of jumping as regarding the size and weight. EPFL presented called EPFL jumper v1(figure 7), EPFL jumper v2 (figure 8)and EPFL jumper v3 .They have all weight between $7 \mathrm{~g}$ and $14.3 \mathrm{~g}$ and are able to jump 27 times their own size.[6].

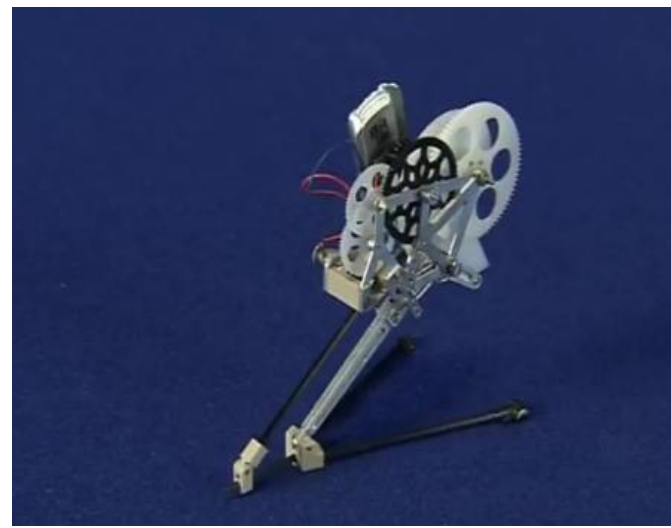

Figure 7: EPFL jumper v1

EPFL developed some small robot in size, remove the obstacles, jumps at low energetic cost. They are able to upright after landing, steer and jump again.[7]. These robots are based on the jumping mechanism of insects link locusts and fleas. After successful of this, they launch new hybrid robot which can glide and jump also.

They add the wings to prolong the jumps. This new robot called EPFL jump glider. (figure 10)It has a mass of $16.5 \mathrm{~g}$ and is able to jump from elevated positions, perform steered gliding flight, land safely and locomotion on ground with repetitive jumps.

EPFL jump glider consists of the gearbox including motor, gearwheels and cam, the main leg, $1.3 \mathrm{~mm}$ carbon rods as feet, the infrared receiver and a $10 \mathrm{mAh}$ Lithium Polymer battery [6]. 


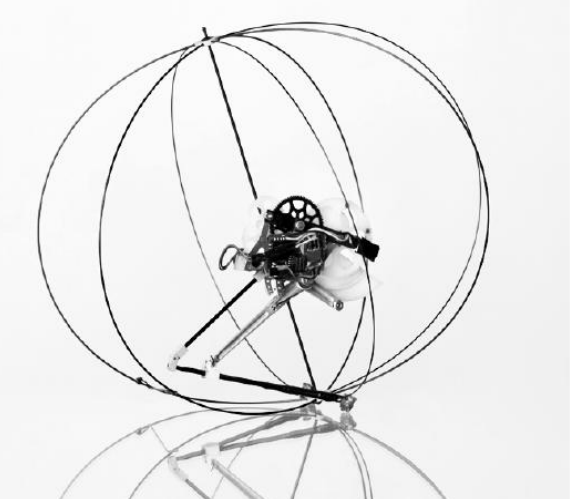

Figure 8: EPFL jumper v2

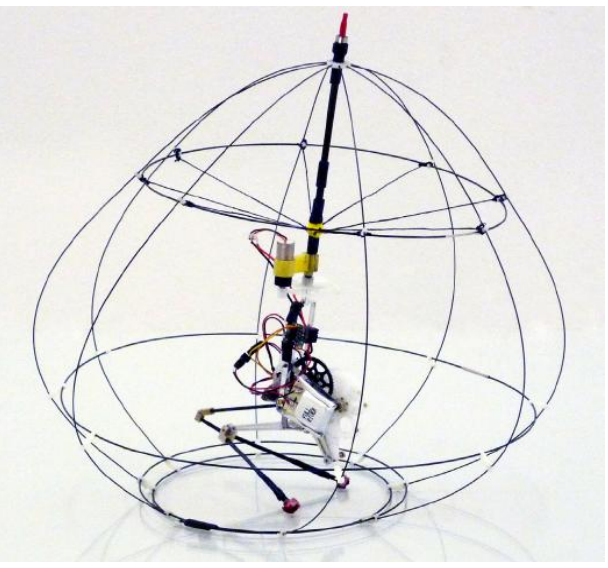

Figure 9: EPFL jumper v3

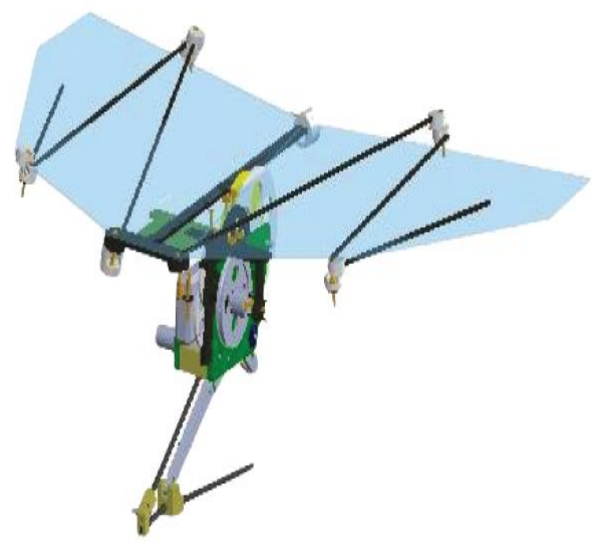

Figure 10:EPFL jumpglider

\section{MINI-WHEGS}

Mini-Whegs ${ }^{\mathrm{TM}}$ are a series of small robots approximately $9 \mathrm{~cm}$ long that use wheel-legs for locomotion .Mini-Whegs inspire by the cockroach and froghopper .Mini-Whegs are robots which abstract insect locomotion, can move over rough terrain an large obstacles. Mini-Whegs have the versions e.g. Mini-Whegs 1(figure 11), Mini-Whegs 2(figure 12), MiniWhegs 3(figure 13), Mini-Whegs 5(14)Mini-Whegs 7(figure 15), Mini-Whegs 8(figure 16) .Now Mini-Whegs 9J(figure 17) is a robust, reliable, inexpensive, and lightweight. MiniWhegs 9J,which includes independent running and jumping capabilities.(figure 18).
It can run at more than three body lengths per second and jump over or onto obstacles almost two body lengths tall[8].

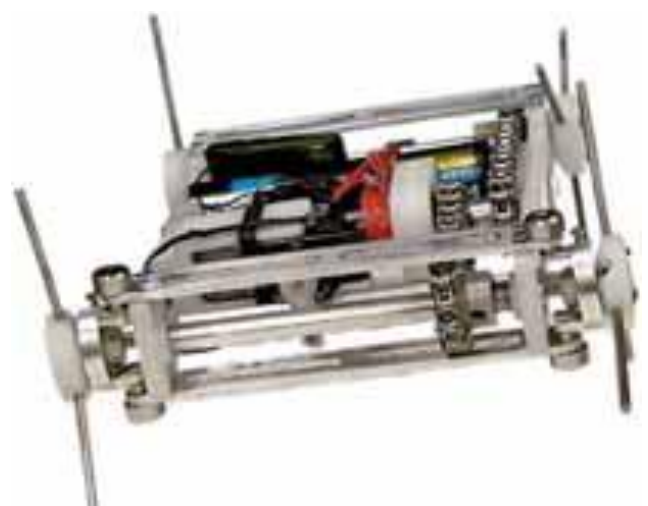

Figure 11: Mini-whegs 1

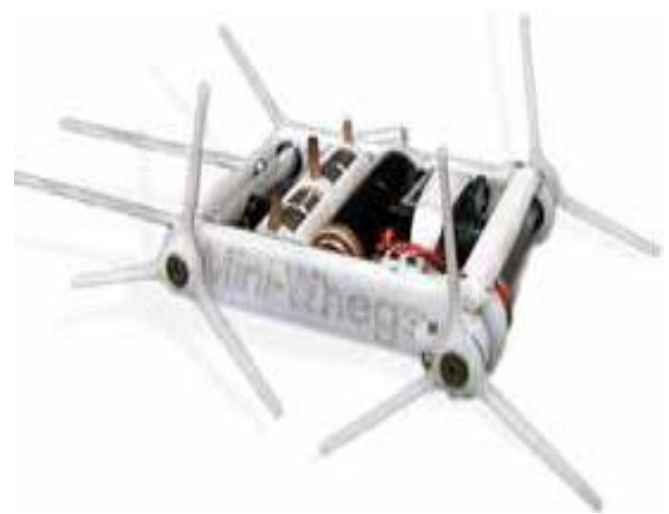

Figure 12: Mini-Whegs 2

Mini-Whegs refer to wheg for locomotion. A wheg is a threespoke appendage driven like a wheel at a central hub. The use of a single large drive motor provides a high power-to-weight ratio, making Whegs highly energetic, and compliant drive components enable passive gait adaptation over irregular terrain.[4]

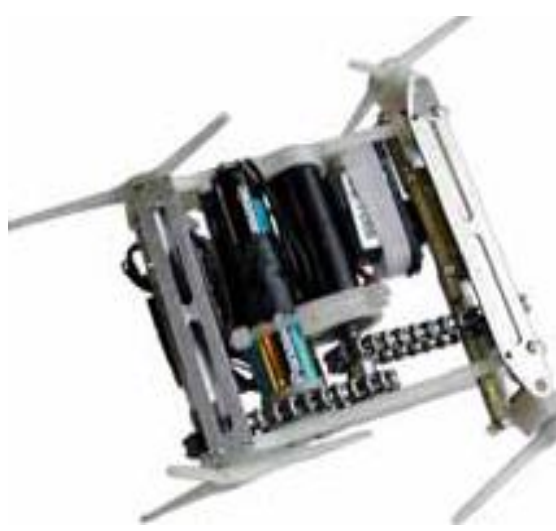

Figure 13: mini-whegs 3 


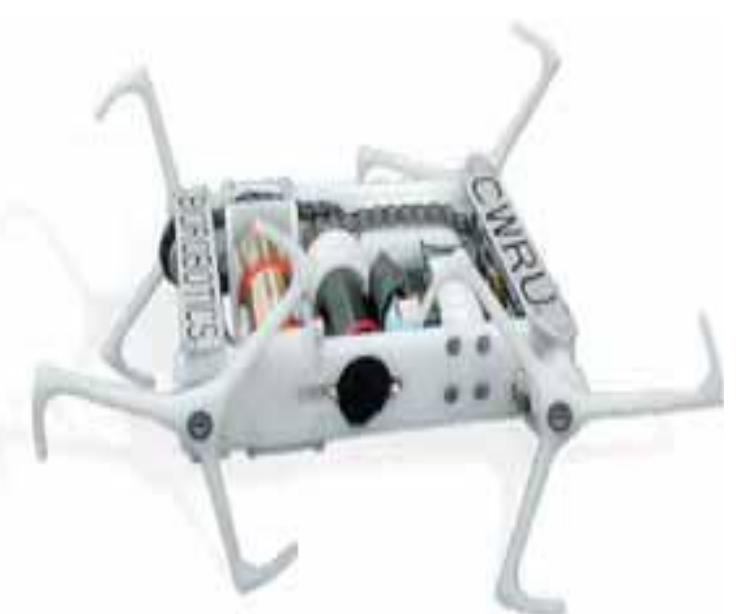

Figure 14: Mini-whegs 5

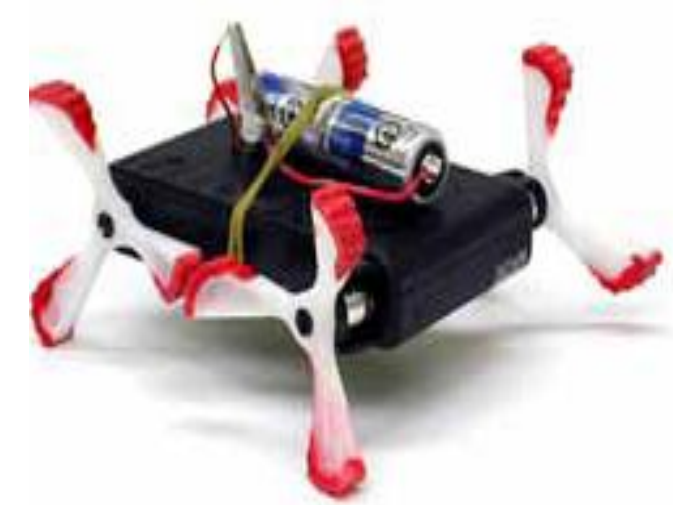

Figure 15: Mini-whegs 7

In Mini-Whegs ${ }^{\mathrm{TM}} 7$ and Mini-Whegs ${ }^{\mathrm{TM}}$ 9J, a 48-pitch nylon rack acts as the control arms to pivot the steering uprights.
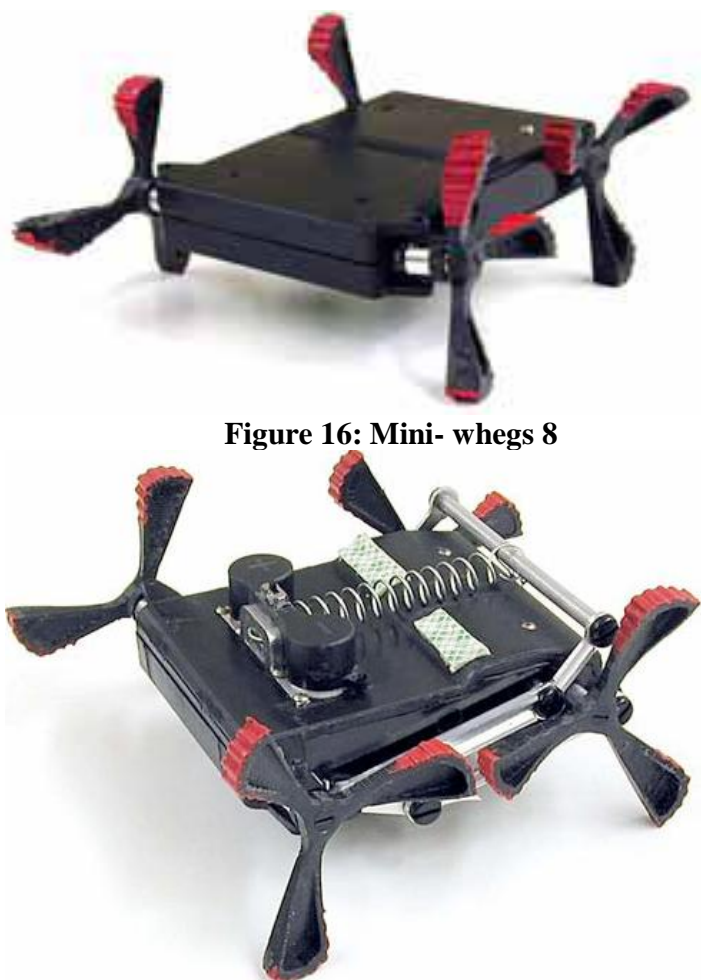

Figure 17: Mini-whegs 9J

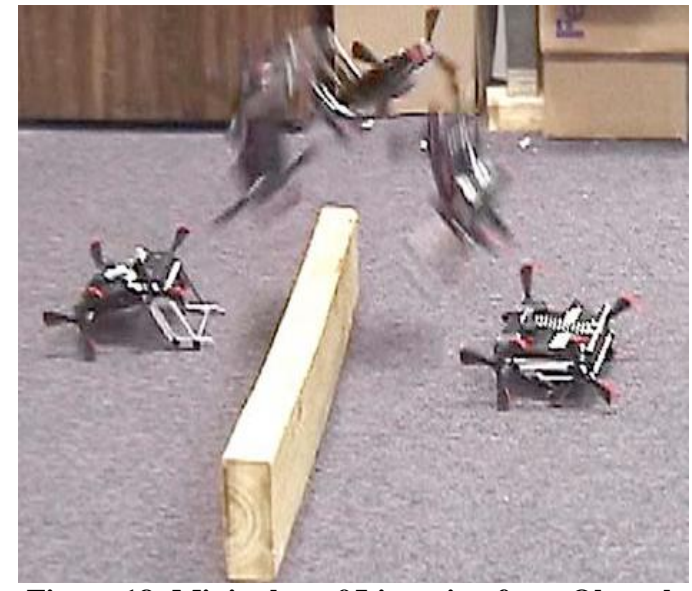

Figure 18: Mini-whegs 9J jumping from Obstacle

\section{CONCLUSION}

To conclude, here we present some miniature bio-inspired robot which enhance the phenomena of jumping locomotion because tiny robot with wheels are not much applicable to move in rough terrain.

These robots are basically adopting the jumping, steering, self -righting because of small size. They solve the problem of less weight and size. These mini robots used as mobile sensors in rough terrain .In future, the work will be on electronic sensing, control system, improving frequency, reaction to environment, implement wireless communication and for long jump use more advance mechanisms.

\section{REFERENCES}

[1] Jianguo Zhao, Ning Xi, Bingtuan Gao, Matt W. Mutka, and $\mathrm{Li}$ Xiao. "Development of a Controllable and Continuous Jumping Robot". $11^{\text {th }}$ IEEE International Conference on Robotics and Automation, Shanghai, China. May 2011, 978-1-61284-380-3.

[2] Umberto Scarfogliero, Cesare Stefanini, Paolo Dario, May,2006. A Bioinspired Concept for High Efficiency Locomotion in Micro Robots: the Jumping Robot Grillo.

[3] Malcolm Burrows. J Exp Biol, 2007. Kinematics of jumping in leafhopper insects.

[4] Bram G. A. Lambrecht, Andrew D. Horchler, and Roger D. Quinn ,C.April, 2005.A Small, Insect-Inspired Robot that Runs and Jumps*.

[5] Fei Li,Gabriella Bonsignori, Umberto Scarfogliero, February 2008.Jumping mini-robot with Bi-inspired legs/

[6] Mirko Kovac," Bio-inspired Jumping Locomotion for Miniature Robotics", Ph.D. dissertation, laboratory of intelligent system, Ecole Polytechnique Fédérale de Lausanne (EPFL).2010

[7] Mirko Kovǎc · Manuel Schlegel · Jean-Christophe Zufferey · Dario Floreano, "Steerable miniature jumping robot" Spring Dec,2009[online]. Avalable:http://dx.doi.org/10.1007/s10514-009- 9173-4

[8] G.A. Lambrecht."'A small, insect-inspired robot that runs and jumps" thesis Master of Science in Engineering , Department of Mechanical and Aerospace Engineering, Case Western Reserve University, January 2005. 